\title{
Research Paper \\ Psychological Effect of Media Use on Mental Health of Older Adults during the COVID-19 Pandemic
}

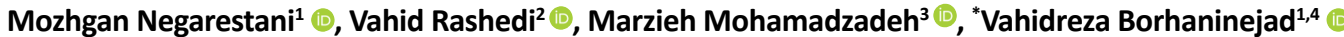

\author{
1. Social Determinants of Health Research Center, Institute for Futures Studies in Health, Kerman University of Medical Sciences, Kerman, Iran \\ 2. School of Behavioral Sciences and Mental Health (Tehran Institute of Psychiatry), Iran University of Medical Sciences, Tehran, Iran. \\ 3. Mane and Semelghan Health Center, North Khorasan University of Medical Science, Bojnurd, Iran. \\ 4. Neuroscience Research Center, Institute of Neuropharmacology, Kerman University of Medical Sciences, Kerman, Iran.
}

\begin{tabular}{|c|c|}
\hline $\begin{array}{l}\text { Use your device to scan } \\
\text { and read the article online }\end{array}$ & Cftrat on: Negarestani M, Rashedi V, Mohamadzadeh M, Borhaninejad V. [Mental Health of Older Adults in the COVID-19 \\
\hline atratas & $\begin{array}{l}\text { Pandemic: The Role of Media Exposure: A Cross-Sectional Study (Persian)]. Iranian Journal of Ageing. 2021; 16(1):74-85. } \\
\text { https://doi.org/10.32598/sija.16.1.1116.6 }\end{array}$ \\
\hline arstrists & doishttps://doi.org/10.32598/sija.16.1.1116.6 \\
\hline
\end{tabular}

\section{(c) (i) (8)}

Received: 11Sep 2020 Accepted: 29 Dec 2020 Available Online: 01 Apr 2021

Key words: Mental health, Older adults, COVID-19, Media

\section{A B STRACT}

Objectives Low media literacy and exposure to media-produced content during the Coronavirus Disease 2019 (COVID-19) pandemic can affect the health of people in the community. This study aims to investigate the psychological effects of media use on the mental health of community-dwelling older adults. Methods \& Materials In this descriptive-analytical study with a cross-sectional design, 200 elderly people aged $\geq 60$ years participated who were selected from daycare rehabilitation centers and retirement centers in Kerman, Iran in 2020 using a convenience sampling method. Data were collected using a demographic form (Surveying age, gender, education, occupation, marital status, income, and media use), and the 12-item General Health Questionnaire.

Results The mean age of participants was $66.60 \pm 5.08$ years. More than three-quarters of them were following the latest COVID-19 news via media (Local radio and television). Most of them (77.4\%) had poor mental health status. Higher educational level $(P<0.001)$, employment $(p=0.003)$, and low frequency of media use $(P<0.001)$ were the protective factors against poor mental health. High-frequency media use was a risk factor for the mental health of older adults during the COVID-19 outbreak.

Conclusion The mental health of community-dwelling older adults during the COVID-19 pandemic is affected by media use and its frequency. It is recommended that older adults should avoid following the latest COVID-19 news, especially those from unreliable sources. Moreover, media managers should have more control over the news related to the COVID-19.

\section{Extended Abstract}

\section{Introduction}

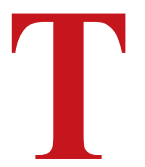

he current Coronavirus Disease 2019 (COVID-19) pandemic has forced the elderly to stay home to be safe from the disease, but various information, some of which are rumors and misinformation, put their mental health at risk and make them anxious and wor- ried. Encountering news related to COVID-19, its speed of transmission, and mortality rate can affect the mental health of people in different societies. The World Health Organization (WHO) has highlighted the role of misinformation and rumors in creating anxiety, worry, and fear, as well as spreading the social stigma of COVID-19. Since proper knowledge of the real functions and impact of the press, radio, television, and cyberspace on the opinions of people especially the elderly, is also very useful for policymakers and governers, this study aims to examine the psychologi-

\section{Corresponding Author:}

Vahidreza Borhaninejad, PhD.

Address: Social Determinants of Health Research Center, Institute for Futures Studies in Health, Kerman University of Medical Sciences, Kerman, Iran. Tel: +98 (34) 31325600

E-mail: v.borhaninejad@kmu.ac.ir 
cal effects of the media on the mental health of communitydwelling elderly people.

\section{Materials and Methods}

This is a descriptive-analytical study with a cross-sectional design conducted in 2020. Participants were 200 older adults aged $\geq 60$ years living in Kerman, Iran. Due to the COVID-19 pandemic and lack of access to the elderly, samples were selected using a convenience sampling method from two daycare rehabilitation centers under the auspices of the Welfare Organization and two pension centers affiliated to the National Association of Retirement Administrators and the Social Security Organization's Association of Retirement Administrators. After receiving the contact numbers of the participants from the mentioned centers and obtaining permission from the relevant authorities, interviews were via phone. The questions were about their demographic information (Age, gender, education, occupation, marital status, and income) as well as three questions to measure the status of media use. The 12-item General Health Questionnaire (GHQ-12) was also used to assess their mental health. Finally, 20 incomplete questionnaires were excluded from the study, and the data of 180 remained questionnaires were analyzed. The collected data were analyzed in SPSS v. 21 software. Due to the normality of data distribution based on the results of the Kolmogorov-
Smirnov test, t-test, one-way ANOVA, and logistic regression analysis were used to analyze the data.

\section{Results}

The mean age of participants was $66.60 \pm 5.08$ years; 134 reported that they follow the news related to COVID-19 via the media (74.5\%). About half of them reported the local media, including radio and television was the most important source of information related to COVID-19 for them (44.8\%) such that most of them used the local media once a day to obtain the information. More than three-quarters of the elderly (77.4\%) had poor mental health status. Findings showed that there was no significant difference in mental health based on gender $(\mathrm{P}=0.5)$ and marital status $(\mathrm{P}=$ $0.46)$. Those with a higher educational level $(\mathrm{P}=0.002)$ as well as employed ones $(\mathrm{P}=0.009)$ had better mental health status. For logistic regression analysis, the effects of confounding factors such as education, employment status, media use, and frequency of media use were controlled. The findings of the final regression model showed that higher educational level, employment, and not using media for getting the latest Covid-19 news were the protective factors against poor mental health during the COVID-19 pandemic. Those with academic education and employed ones were $50 \%$ and $30 \%$ less likely to have poor mental health than others $(\mathrm{P}<0.001$ and 0.003 , respectively). In those who used the media, the likelihood of having poor mental health

Table 1. Results of logistic regression analysis for examining the effects of study variables on mental health of older adults

\begin{tabular}{|c|c|c|c|}
\hline \multicolumn{2}{|c|}{ Variable } & \multirow[t]{2}{*}{ Adjusted Odds Ratio (95\%Cl) } & \multirow[t]{2}{*}{$\mathbf{P}$} \\
\hline \multirow{4}{*}{ Education } & Illiteratea & & \\
\hline & Without high school diploma & $0.87(0.33-1.56)$ & 0.09 \\
\hline & With high school diploma & $0.610(0.12-0.88)$ & 0.001 \\
\hline & Academic & $0.508(0.117-0.93)$ & $<0.001$ \\
\hline \multirow[b]{2}{*}{ Occupation } & Unemployeda & & \\
\hline & Employed & $0.701(0.210-0.80)$ & 0.003 \\
\hline \multirow{3}{*}{ Media use } & No & & \\
\hline & & & \\
\hline & Yes & $2.10(1.12-3.95)$ & $<0.001$ \\
\hline \multirow{4}{*}{ Frequency of media use } & Weekly or morea & & \\
\hline & Once every few days & $1.15(0.55-1.80)$ & 0.07 \\
\hline & Once a day & $2.12(1.55-3.5)$ & 0.008 \\
\hline & Several times a day & $3.04(1.98-4.02)$ & $<0.001$ \\
\hline
\end{tabular}


was two times higher than in non-users $(\mathrm{P}<0.001)$. On the other hand, those who used the media several times a day to get the news related to COVID-19 were three times more likely to have poor mental health than those who rarely followed the news and information (Table 1).

\section{Discussion and Conclusion}

The mental health of community-dwelling elderly people during the COVID-19 pandemic is affected by their media use and its frequency. In this regard, it is suggested that the elderly, while increasing their media knowledge, avoid following the news related to COVID-19, especially the news from unreliable sources as much as possible. They should receive information from reliable sources and share it after ensuring their accuracy. They should focus more on information related to disease prevention and how to deal with self-quarantine conditions to promote mental health. Media managers also need to monitor and review the COVID-19-related news considering their effect on the mental health of people, especially the elderly.

\section{Ethical Considerations}

Compliance with ethical guidelines

Ethical approval was obtained from the Institutional Review Board of Kerman University of Medical Sciences (Code: IR.KMU.REC.1398.739).

\section{Funding}

This study was financially supported by the Deputy for Research of the Kerman University of Medical Sciences.

\section{Authors' contributions}

Conceptualization, supervision, methodology: Mozhgan Ngarestani and Vahidreza Borhaninejad; writing-original draft, investigation: All authors; writing- editing \& review, funding acquisition, resources: Vahidreza Borhaninejad.

\section{Conflicts of interest}

The authors declared no conflict of interest. 


\title{
سلامت روان سالمندان در پاندمى كوويد 19 (: نقش مواجهه با رسانهها
}

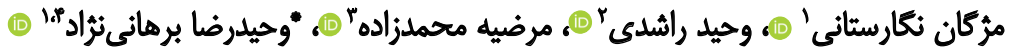

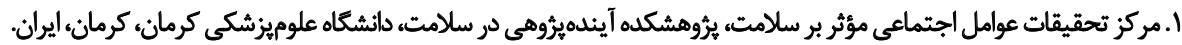

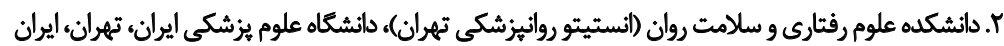

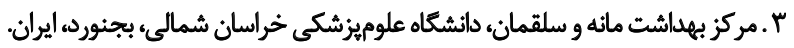

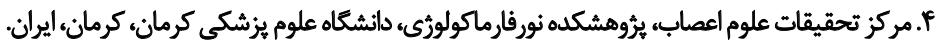

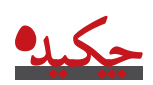

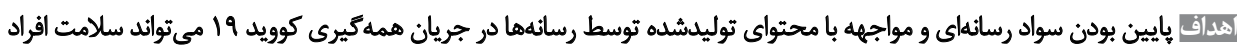

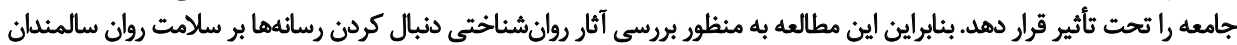
مقيم جامعه صورت كرفت.

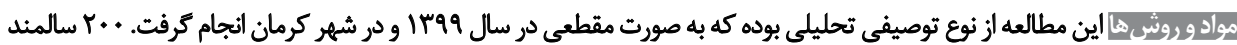

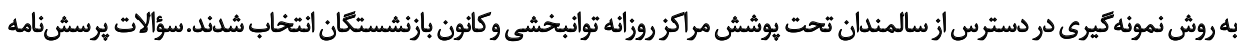

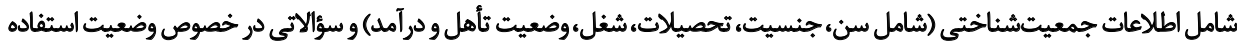

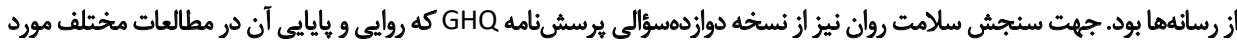

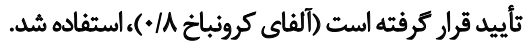

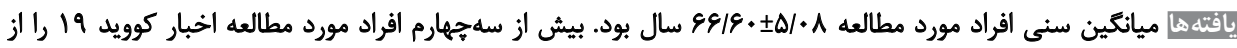

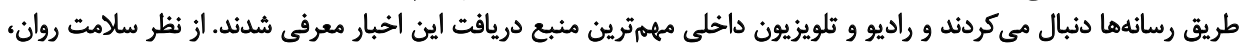

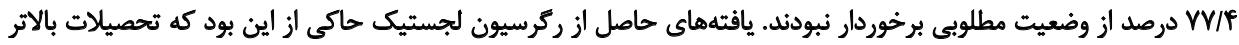

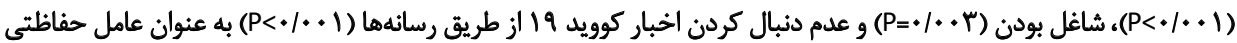

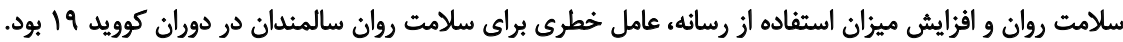

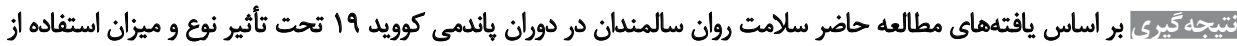

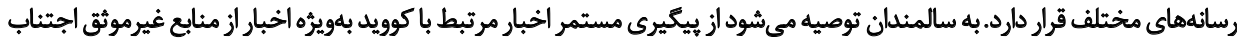

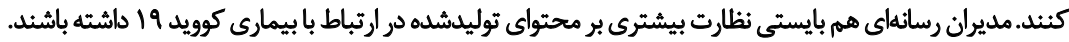

تاريخ دريافت: آ شهريور

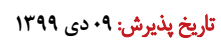

تاريخ انتشار: Tا فروردين

\section{كليدوارّها: \\ سلامت روان، سالمند،كوويد 19. - سلامت رسانه}

بسيارى از بيمارىهاى روانشناختى و مشكلات سلامت روان رات

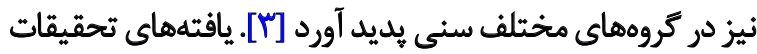

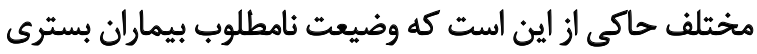

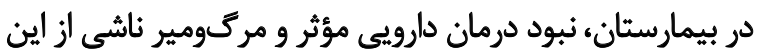

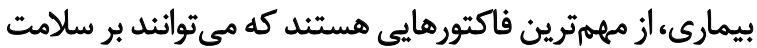

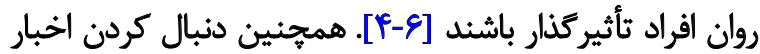

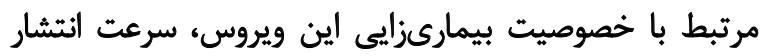

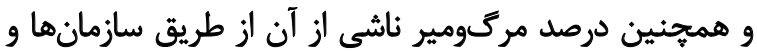

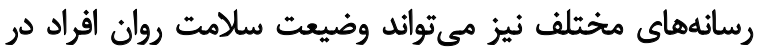

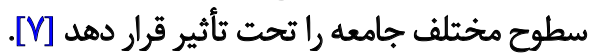

اواخر سال 19 • ب ميلادى انتشار يك بيمارى ويروسى در شيهر

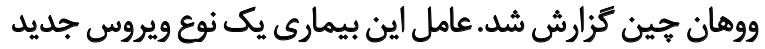

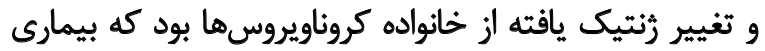

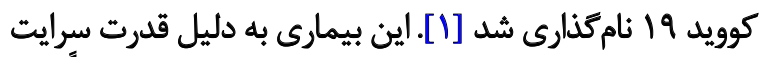

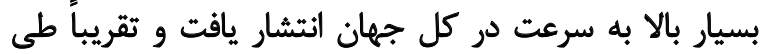

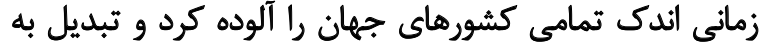

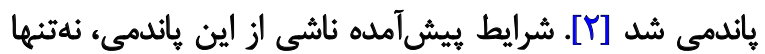

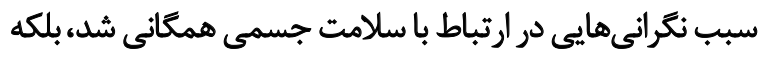

"نويسنده مسئول:

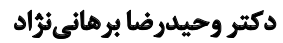

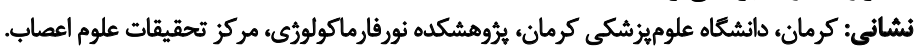

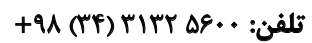

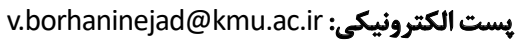


در مورد بالا رفتن موارد مركومير و اين موضوع كه سالمندان

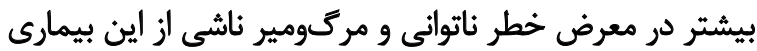

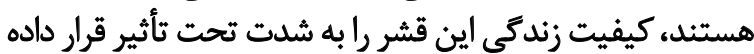

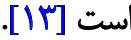

متأسفائه در ياندمى ها به دليل كسترش سريع بيمارى و وجود

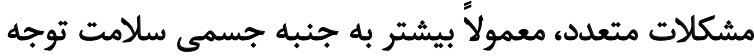

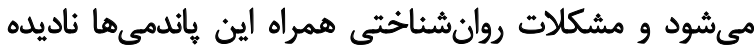

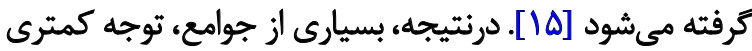

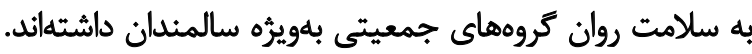

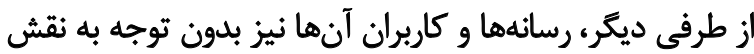

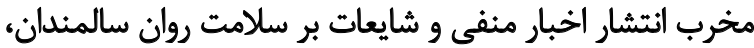

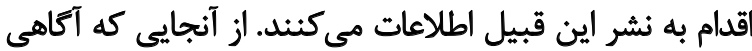

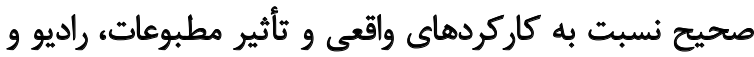

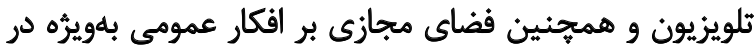

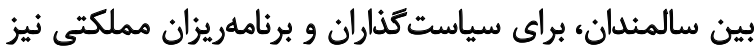

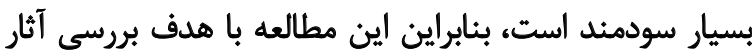
روانشناختى دنبال كردن رسائهها بر سلامت روان سائن سالمندان

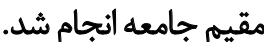

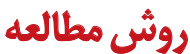

اين مطالعه از نوع توصيفى تحليلى است كه به صورت مقطعى

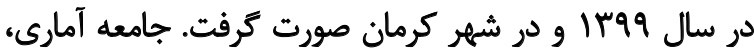

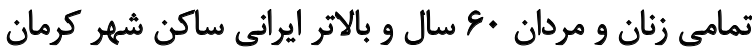

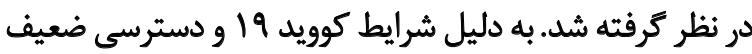

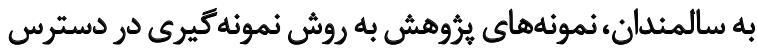

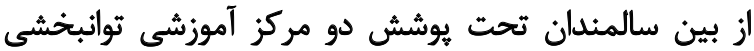

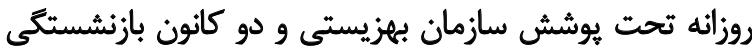

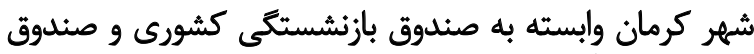
بازنشستكى تأمين اجتماعى انتخاب شدند. بإندي

جمع آورى دادهها از اواسط خرداد شروع شد و تا اواخر مرداد

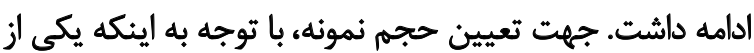

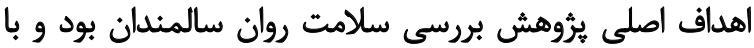

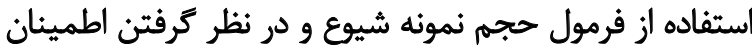

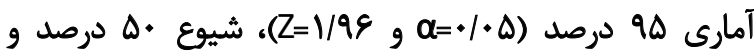

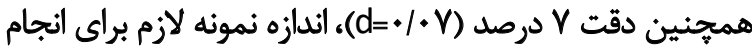

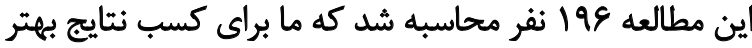
حجمى معادل ·. - نفر لحاظ كرديم.

با توجه به وضعيت شيوع بيماري كوويد 19 و لزوم رومايت روايت

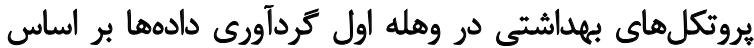

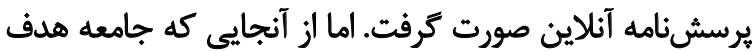

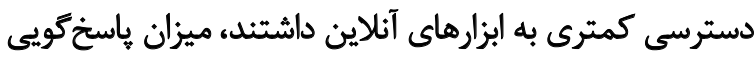

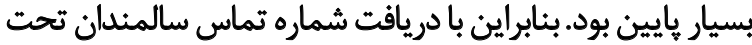
يوشش مراكز مذكور و كسب مجوز از مسئولين مربوطه، از طريق بايق
سازمانهاى رسمى و غيررسمى زيادى در دوران هاندمى إنى

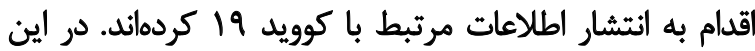

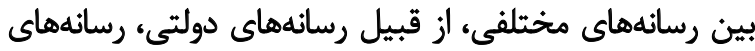

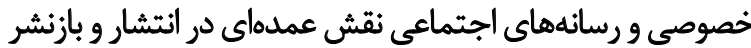

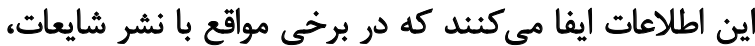

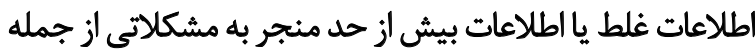

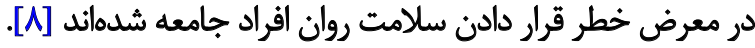

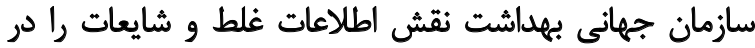

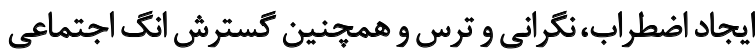

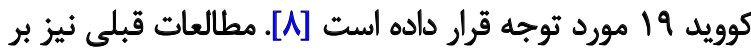

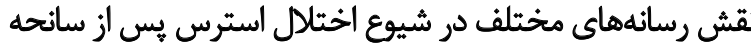

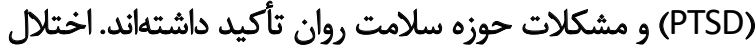

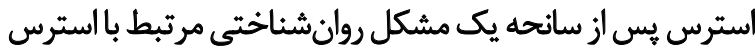

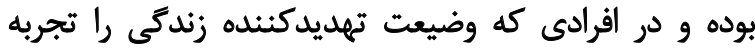

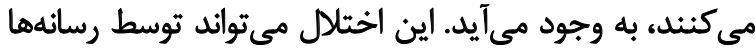

تشديد شود [• [9:

نظريههاي متعددى به بررسى نقش رسانهها بر افكار عمومى

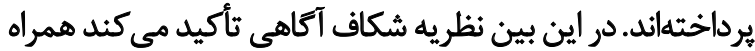

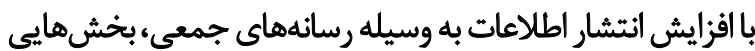

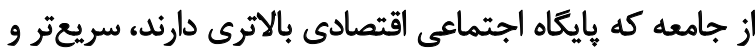

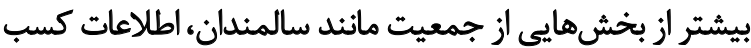

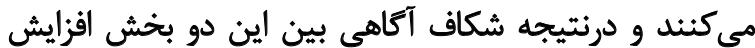

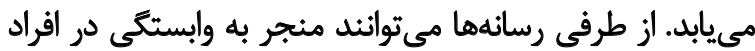

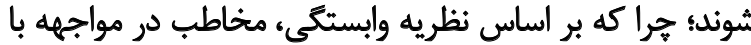

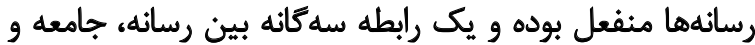

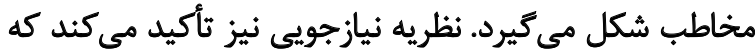

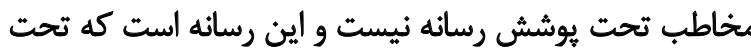

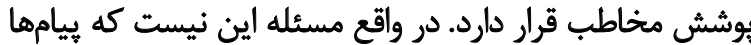

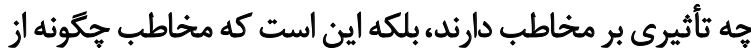

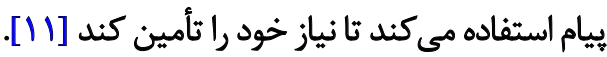
بر اين اساس ترويج رفتارهاى ارتقادهئده سلامت روان در بردي

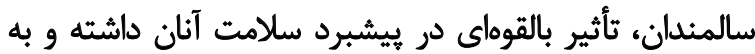

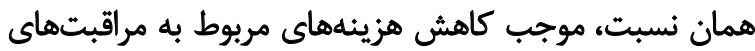

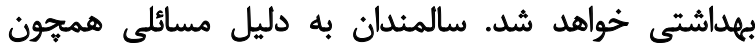

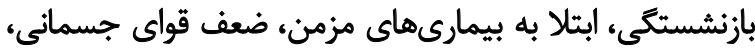

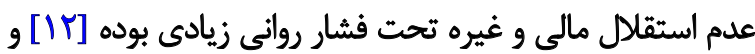

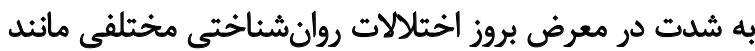

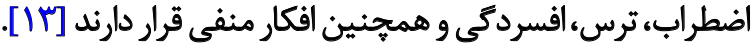

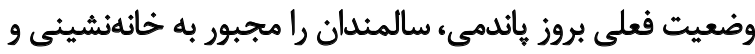

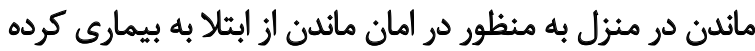

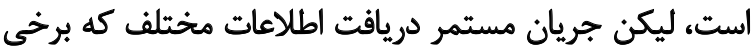

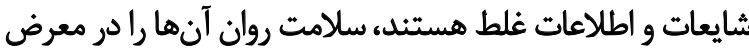

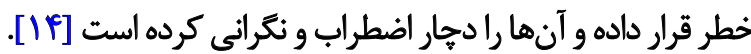

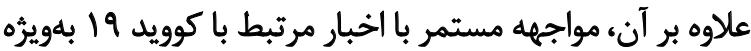


مورد استفاده (اجتماعى، داخلى نظير راديو و تلويزيون دولتي،

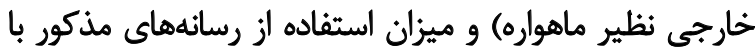

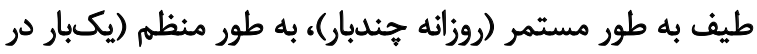

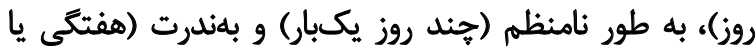

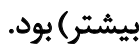

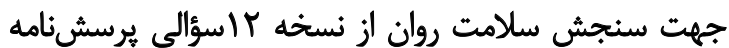

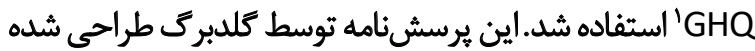

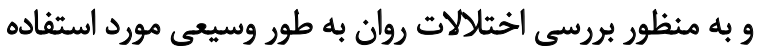

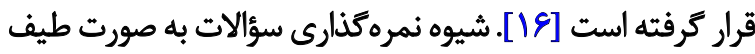

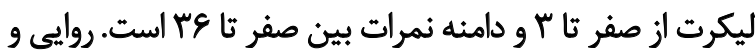

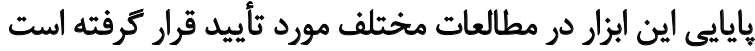

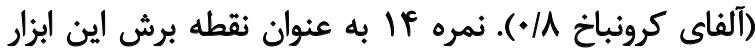

$$
\text { تعيين شده است كرون [IV] }
$$

درنهايت، بيست برسشنامه به دليل نواقص از تحقيق خارج

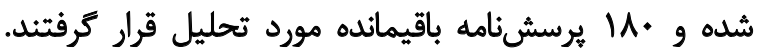

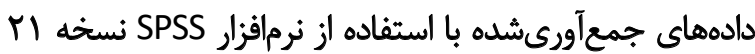

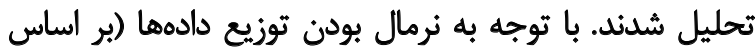

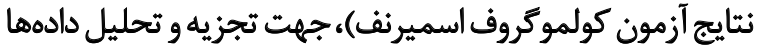

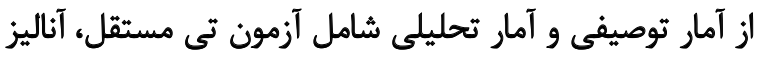
واريانس يكطرفه و ركرسيون لجستيك استفاده شد.

ياقتهها

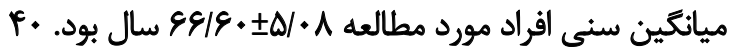

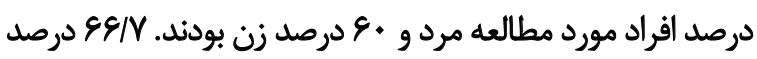

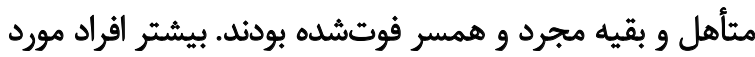

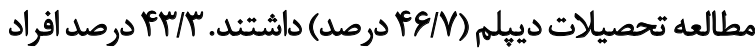

1. General Health Questionnaire-12
تلفن ثابت يرسشكرى صورت كرفت. ليست افراد تحت يوشش مرأ

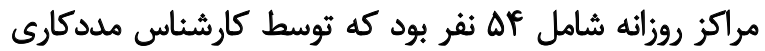

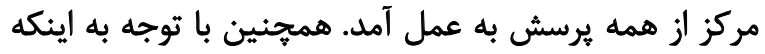

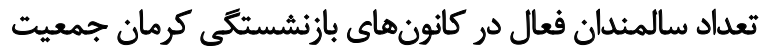

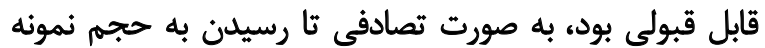

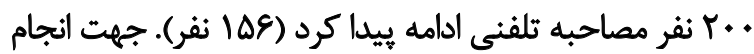

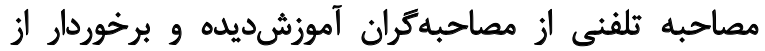
مهارتهاى برقرارى ارتباط با سالمندان استفاده شد. ابتدا از إفراد إداد

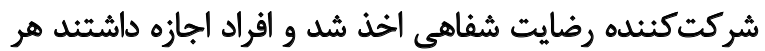

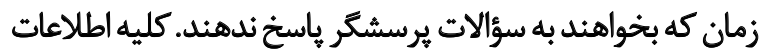

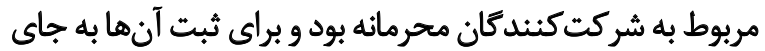

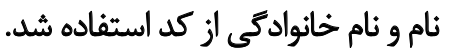

سؤالات مصاحبه از اطلاعات جمعيتشناختى شامل سن،

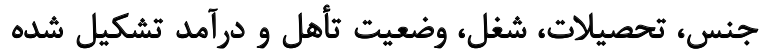

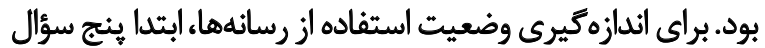

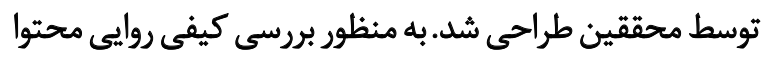

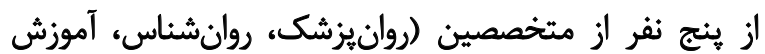

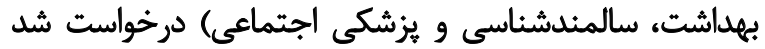

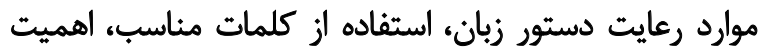

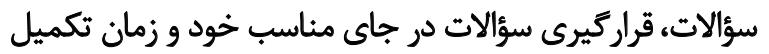

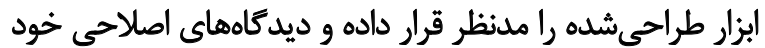

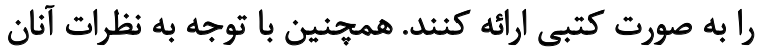

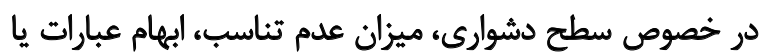

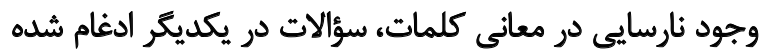

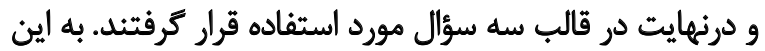

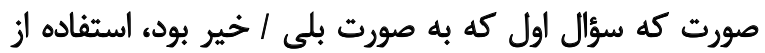

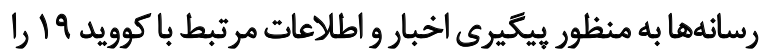

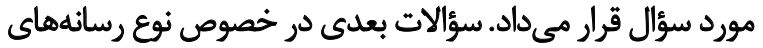

جدول ا. ميزان استفاده از رسانهاي مختلف به منظور دريافت اخبار و اطلاعات كوويد 19

\begin{tabular}{|c|c|c|c|c|c|}
\hline \multicolumn{5}{|c|}{ ميزان استفاده (درصد) } & \multirow{2}{*}{ ثوع ربانه } \\
\hline جمع كل & هفتكَى يا بيشُتر & هند روز يكبار & روزى يكبار & روزى جندبار & \\
\hline$r \Delta(T / N)$ & 1. (TNE) & $M(M / M)$ & $\Lambda(T / A)$ & $\Delta(1 F / M)$ & رسائهاى اجتماعى \\
\hline G. (fF/A) & $8(1 * / *)$ & If $(\pi / T)$ & $\mu+(\Delta+/ *)$ & $1 \cdot(1 \& / V)$ & رسانههاى داخلى \\
\hline$r r(I V / T)$ & $\Delta(M / N)$ & $g(4 / N)$ & $\Lambda(M+/ \Lambda)$ & $f(I V / f)$ & رسانههاى خارجى \\
\hline $18(11 / 9)$ & $r(I T / \Delta)$ & $f(T \Delta / \cdot)$ & $\Lambda(\Delta \cdot / \cdot)$ & $r(I T / \theta)$ & جند رسائه \\
\hline ine & r & re & $\Delta f$ & $r$ & كل \\
\hline
\end{tabular}


جدول r. رابطه بين متغيرهاى جمعيتشناختى و استفاده از رسائه با سلامت روان در افراد موردمطالعه

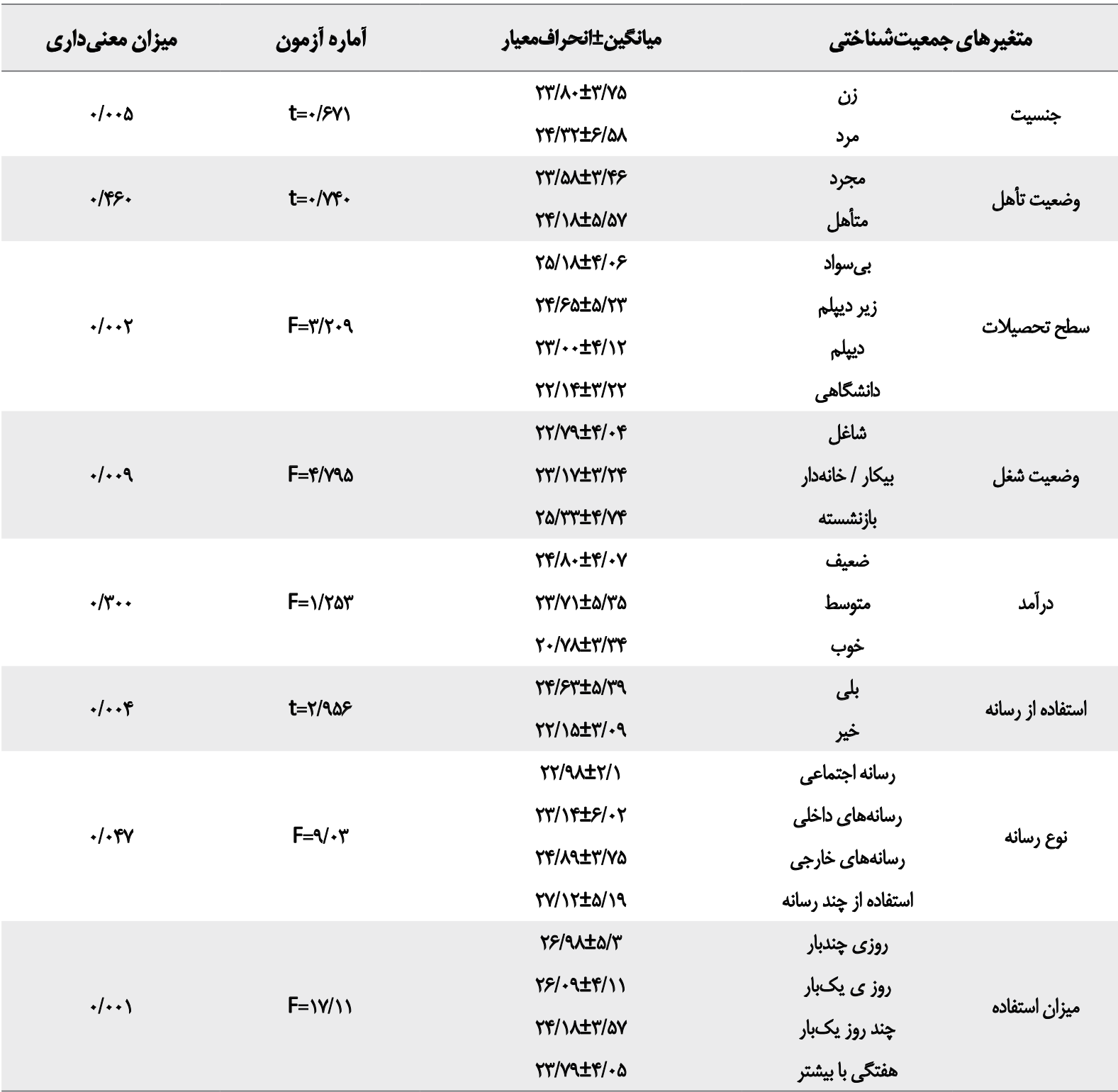

\section{L}

بر اساس نتايج، بيش از سهجهارم از سالمندان موردمطالعه

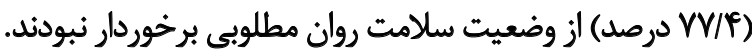

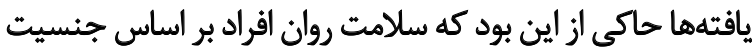

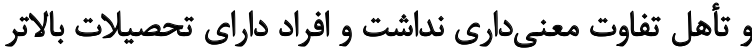

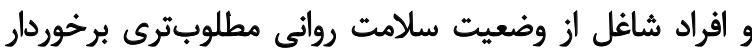

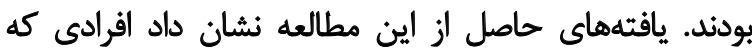

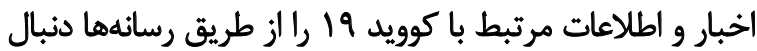

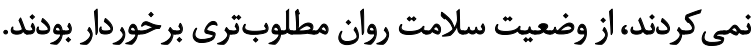

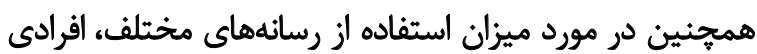

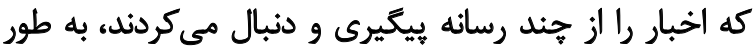

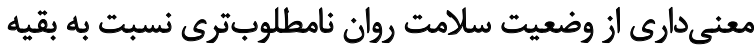

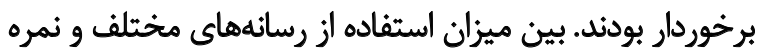

بازنشسته و بقيه افراد شاغل يا خانهدار بودند. بيشتر سالمندان

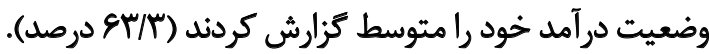

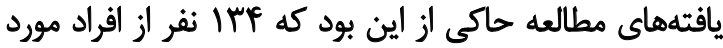

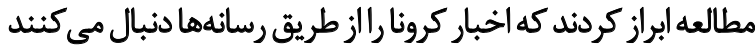
Vر VF/Q)

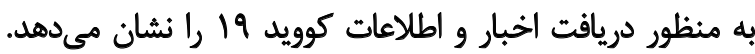

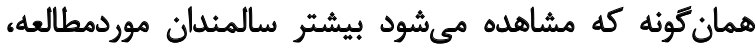

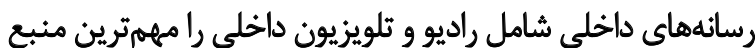

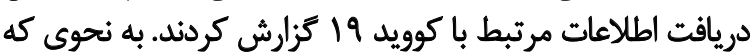

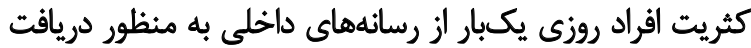
اطلاعات مرتبط با بيمارى كوويد 19 استفاده كردهاند. 
جدول س. نتايج حاصل از ركر سيون لجستيك در خصوص سلامت روان بر حسب متغيرهاى مستقل

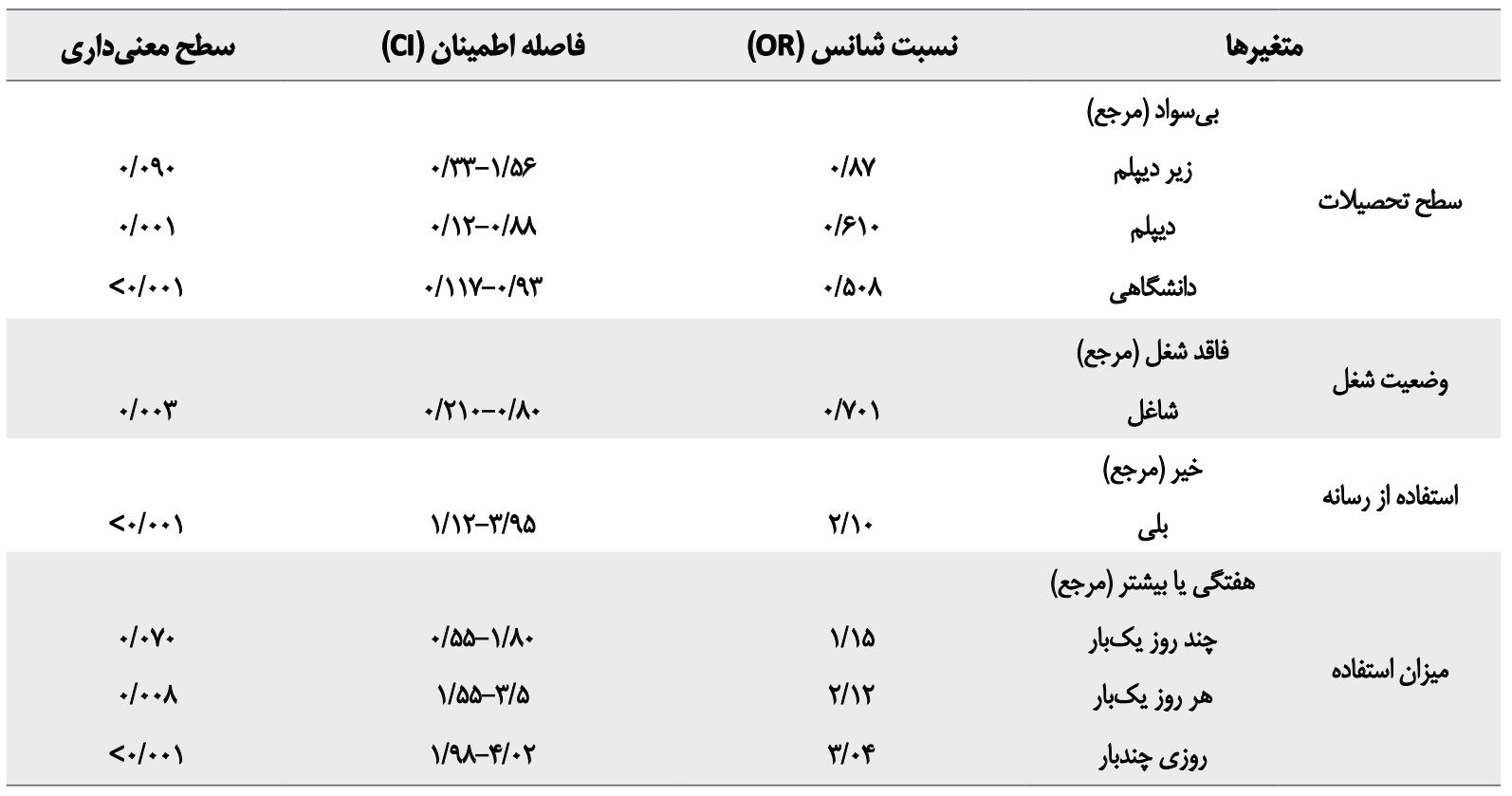

L

افراد جامعه، بلويزه سالمندان ايجاد شده است. فارغ از مشكلات

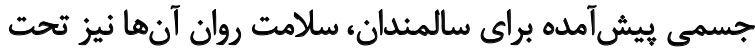

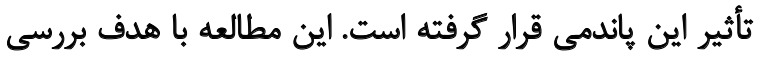

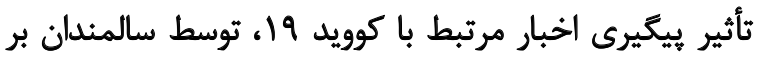
سلامت روان آنها انجام شد.

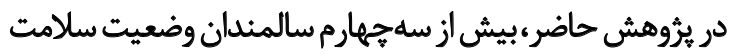

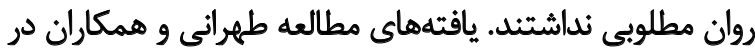

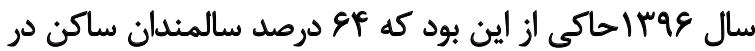

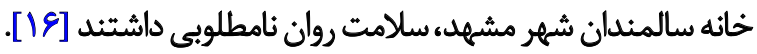

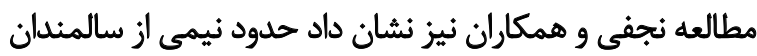

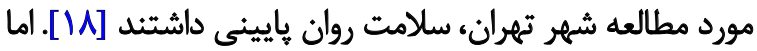

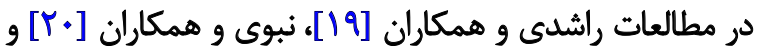

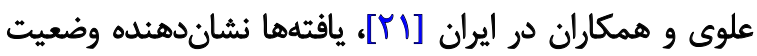

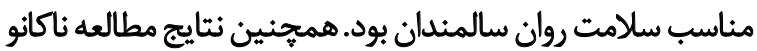

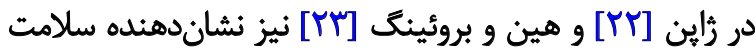

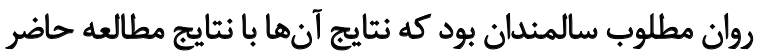

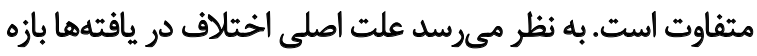

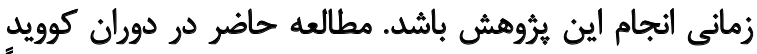

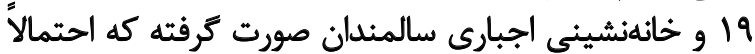

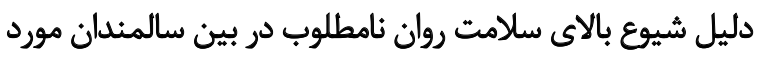

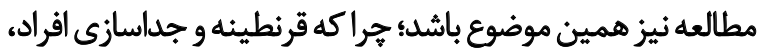

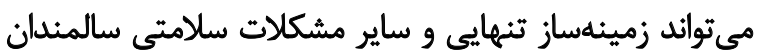

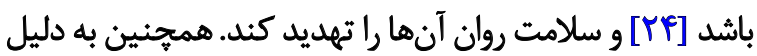

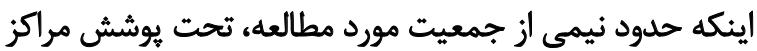
روزانه سالمندان بودند و تا قبل از زمان شيوع كوريد 9 الز ازئ خدمات
سلامت روان رابطه معنى دارى مشاهده شد. به نحوى كئ كه افراد

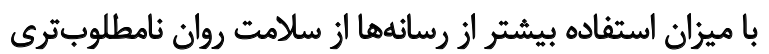

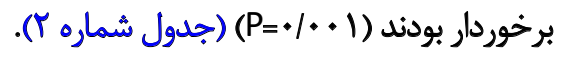
در تحليل ركرسيون لجستيك جهت برآورد اثر متغير اصلى دوري

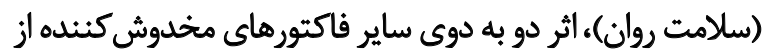

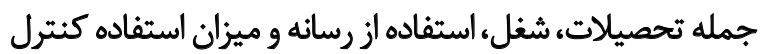

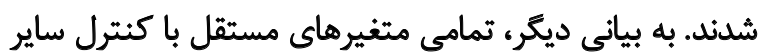

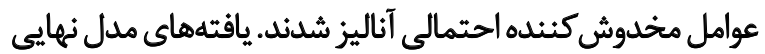

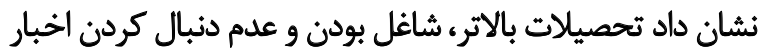

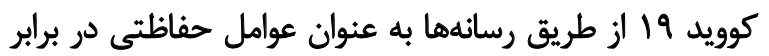

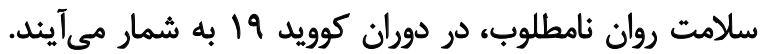

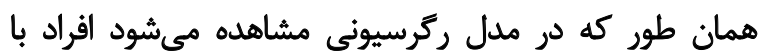

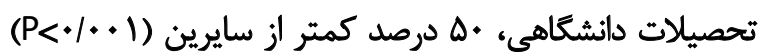

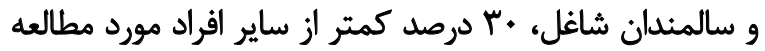

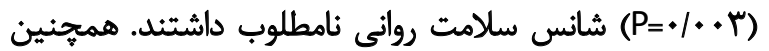

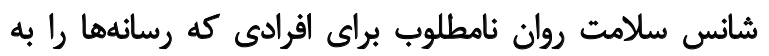

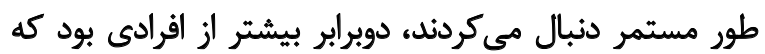

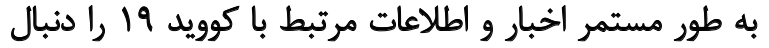

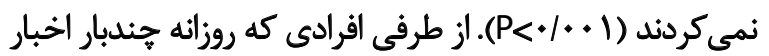

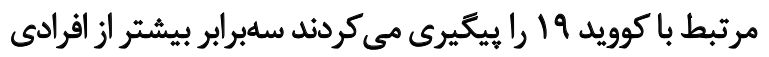

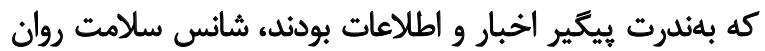
نامطلوب داشتند (جدول شماره ب). بحث همززمان با ياندمى كوويد 19 مشكلات متعددى براى تمام 


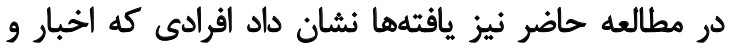

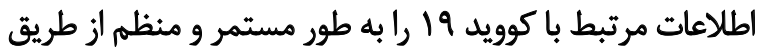

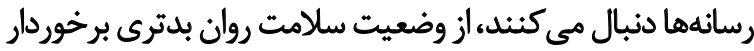

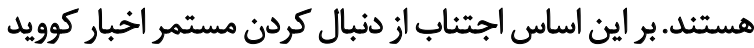

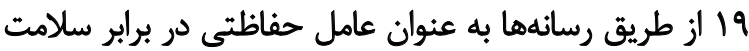

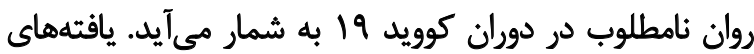

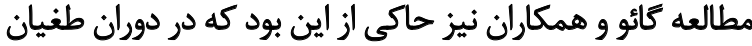

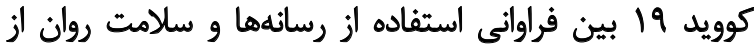

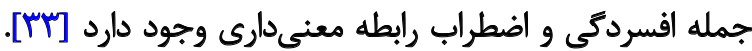

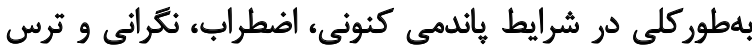

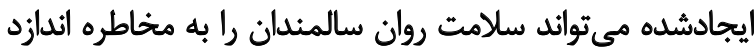

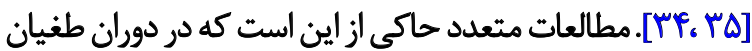

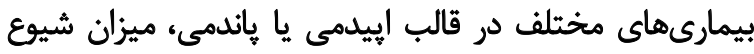

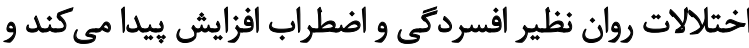
اين وضعيت تحت تأثير انتشار اطلاعات مختلف و و وسيع، مي توتواند

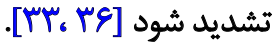

در جهان امروز تأثيركذارى رسانهها بر جامعه غير قابل انكار

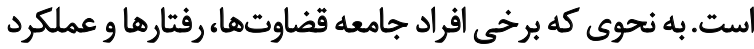

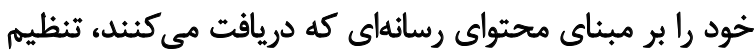

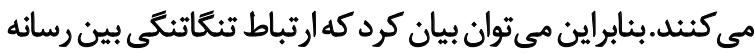

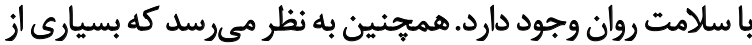

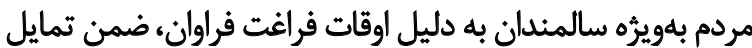

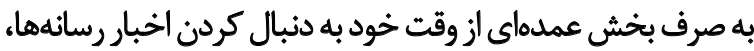

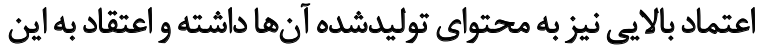

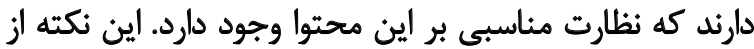

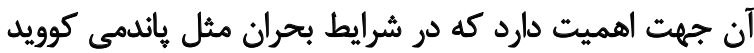

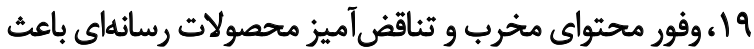

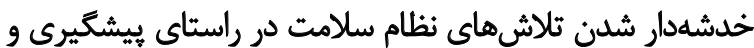

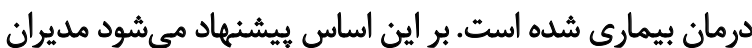

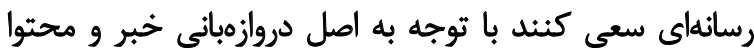

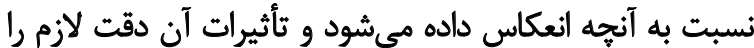

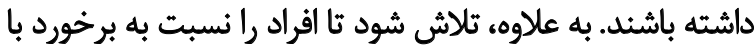

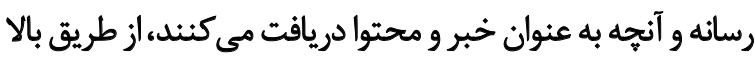

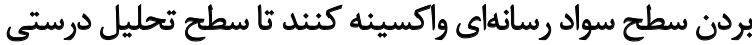

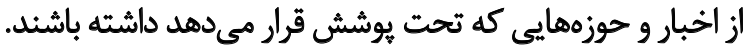

\section{نتيجلة تيرى نهايى}

بر اساس يافتههاى مطالعه حاضر سلامت روان سالمندان

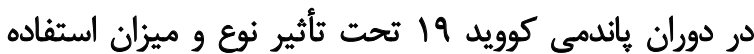

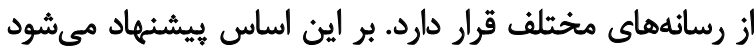

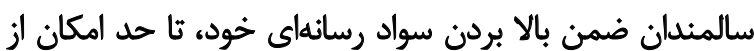

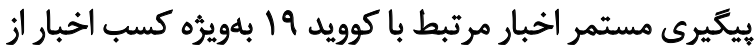

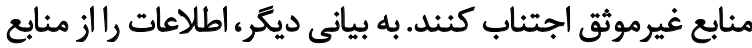

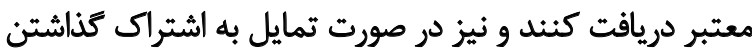

برنامههاى حضورى و جمعى اين مراكز استفاده مي كردند، ممكن

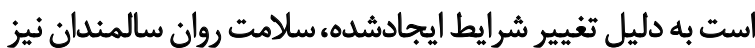
دجار هالش شده باشد.

در اين مطالعه بين سلامت روان سالمندان با ثأهل و جنسيت

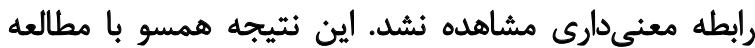

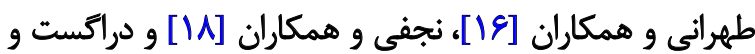

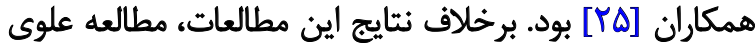

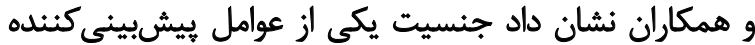

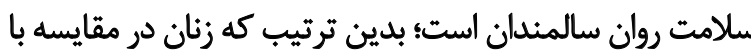

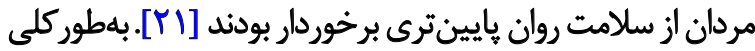

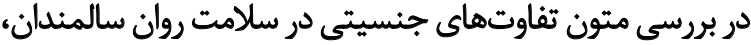

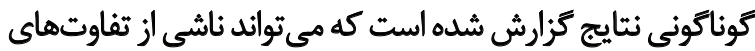

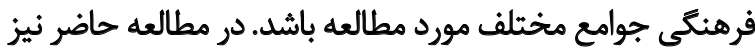

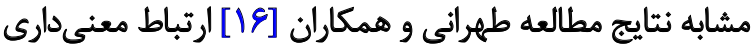

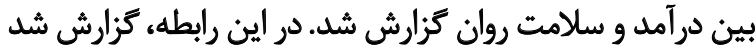

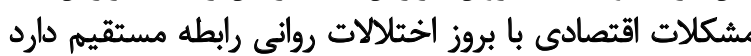

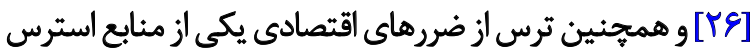

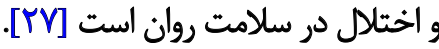

يافتههاى مطالعه حاضر حاكى از اين بود كه تحصيلات از عوامل

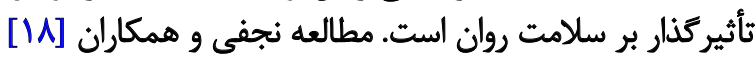

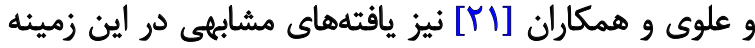

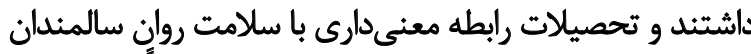

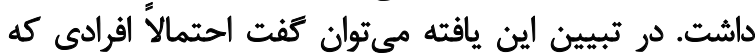

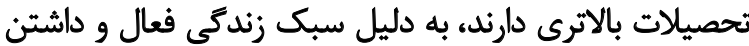

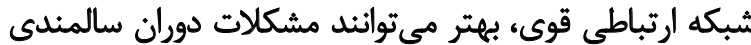

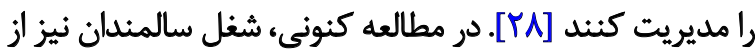

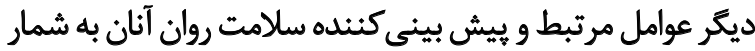

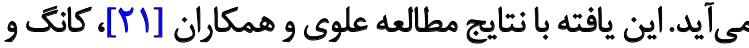

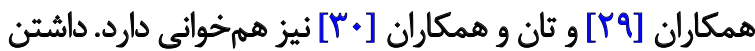

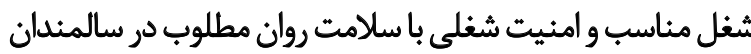

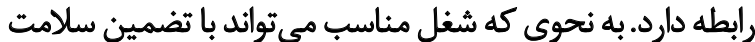

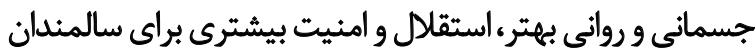

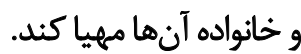

در اين مطالعه بيش از سهجهارم سالمندان اطلاعات و اخبار

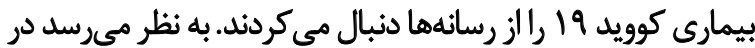

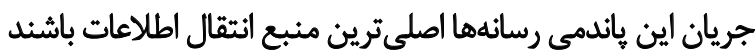

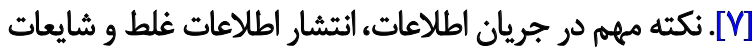

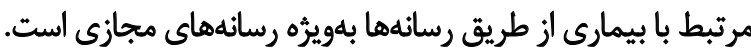

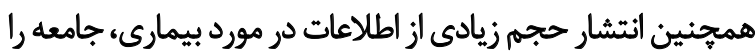

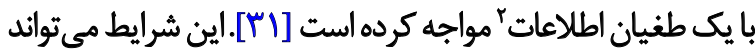
منجر به كاهش سلامت روان افراد جامعه شود [بكان. 
بعد از اطمينان از صحت محتواى دريافتى، اطلاعات رانشر دهند.

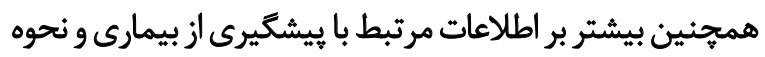

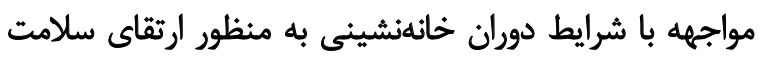

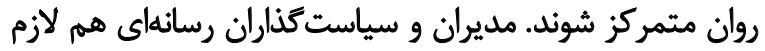
است محتواى توليدشده در زمينه كوويد

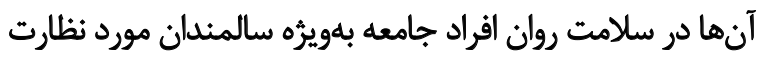

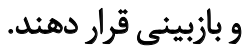

انجام مطالعات طولى و كوهورت با حجم نمونه وسيع به منظور

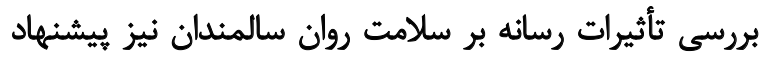

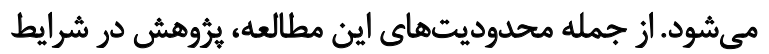

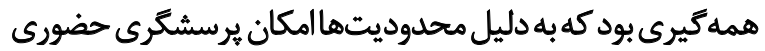

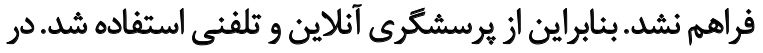

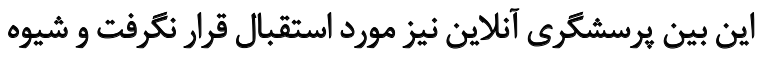

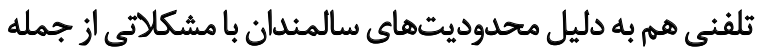

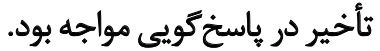

مالاحظات اخلاقى - ماتى

يميروى از اصول الخاق بئوهش

اين برثوهش توسط كميته اخلاق در برثوهش دانشكاه

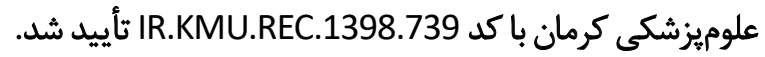

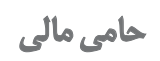

اين بروهش با حمايت مالى مركز تحقيقات عوامل اجتماعى برائ

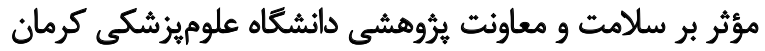

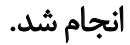

$$
\text { مشاركت نويسند مَان }
$$

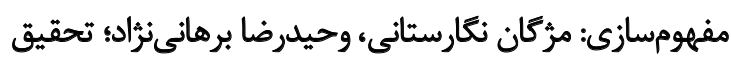

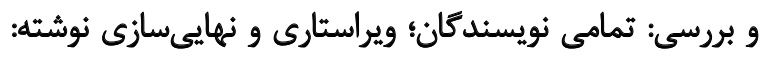
وحيدرضابرهانىنزراد.

$$
\text { تعارض مناقع }
$$

بنابر اظهار نويسندگان اين مقاله هيجگونه تعارض منافعى

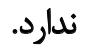




\section{References}

[1] Zhu H, Wei L, Niu P. The novel coronavirus outbreak in Wuhan, China. Global Health Research and Policy. 2020; 5:6. [DOI:10.1186/s41256-020-00135-6] [PMID] [PMCID]

[2] Remuzzi A, Remuzzi G. COVID-19 and Italy: What next? The Lancet. 2020; 395(10231):1225-8. [DOI:10.1016/S0140 6736(20)30627-9]

[3] Shahyad S, Mohammadi MT. [Psychological impacts of Covid-19 outbreak on mental health status of society individuals: A narrative review (Persian)]. Journal of Military Medicine. 2020 22(2):184-92. [DOI:10.30491/JMM.22.2.184]

[4] Xiang YT, Yang Y, Li W, Zhang L, Zhang Q, Cheung T, et al. Timely mental health care for the 2019 novel coronavirus outbreak is urgently needed. The Lancet Psychiatry. 2020; 7(3):228-9. [DOI:10.1016/S2215-0366(20)30046-8]

[5] Lima CKT, de Medeiros Carvalho PM, Lima IDAS, de Oliveira Nunes JVA, Saraiva JS, de Souza RI, et al. The emotional impact of Coronavirus 2019-nCoV (new Coronavirus disease). Psychiatry Research. 2020; 287:112915. [DOI:10.1016/j.psychres.2020.112915] [PMID] [PMCID]

[6] Bo HX, Li W, Yang Y, Wang Y, Zhang Q, Cheung T, et al. Posttraumatic stress symptoms and attitude toward crisis mental health services among clinically stable patients with COVID-19 in China. Psychological Medicine. 2020; 51(4):1-2. [DOI:10.1017/ S0033291720000999] [PMID] [PMCID]

[7] Bao Y, Sun Y, Meng S, Shi J, Lu L. 2019-nCoV epidemic: Address mental health care to empower society. The Lancet. 2020; 395(10224):e37-8. [DOI:10.1016/S0140-6736(20)30309-3]

[8] World Health Organization. COVID-19 Public Health Emergency of International Concern (PHEIC) Global research and innovation forum [Internet]. 2020 [Updated 2020 February 12]. Available from: https://www.who.int/publications/m/item/ covid-19-public-health-emergency-of-international-concern(pheic)-global-research-and-innovation-forum

[9] Neria Y, Sullivan GM. Understanding the mental health effects of indirect exposure to mass trauma through the media. JAMA. 2011; 306(12):1374-5. [DOI:10.1001/jama.2011.1358] [PMID] [PMCID]

[10] Choi DH, Yoo W, Noh GY, Park K. The impact of social media on risk perceptions during the MERS outbreak in South Korea. Computers in Human Behavior. 2017; 72:422-31. [DOI:10.1016/j. chb.2017.03.004] [PMID] [PMCID]

[11] Vindal S. Application of communication theories [Dehghan A, Persian trans]. Tehran: Jameh Shenasan; 2008.

[12] Rhee MK, Mor Barak ME, Gallo WT. Mechanisms of the effect of involuntary retirement on older adults' self-rated health and mental health. Journal of Gerontological Social Work. 2016; 59(1):35-55. [DOI:10.1080/01634372.2015.1128504] [PMID]

[13] Philip J. Impact of COVID-19 on mental health of the elderly. International Journal of Community Medicine and Public Health 2020; 7(6):2435. [DOI:10.18203/2394-6040.ijcmph20202513]

[14] Armitage R, Nellums LB. COVID-19 and the consequences of isolating the elderly. The Lancet Public Health. 2020; 5(5):e256. [DOI:10.1016/S2468-2667(20)30061-X]

[15] Girdhar R, Srivastava V, Sethi S. Managing mental health issues among elderly during COVID-19 pandemic. Journal of Geriatric Care and Research. 2020; 7(1):32-5. https:/ /d1wqtxts1xzle7. cloudfront.net/63475639/JGCR_2020_7_120200530-128369-1
[16] Tehrani H, Vahedian Shahroodi M, Fadayevatan R, Abusalehi A, Esmaeili H. [Mental health status and its related factors in elderly people residing in nursing homes of Mashhad, Iran (Persian)]. Health and Development Journal. 2017; 6(3):171-81. http:/ / eprints.nums.ac.ir/339/1/

[17] Namjoo S, Shaghaghi A, Sarbaksh P, Allahverdipour H, Pakpour AH. Psychometric properties of the General Health Questionnaire (GHQ-12) to be applied for the Iranian elder population. Aging \& Mental Health. 2017; 21(10):1047-51. [DOI:10.1080/1360 7863.2016.1196337] [PMID]

[18] Najafi B, Arzaghi M, Fakhrzadeh H, Sharifi F, Shoaei S, Alizadeh $\mathrm{M}$, et al. [Mental health status and related factors in aged population: Urban health equity assessment and response tool (Urban-HEART) study in Tehran (Persian)]. Iranian Journal of Diabetes and Metabolism. 2013; 13(1):62-73. http://ijdld.tums. ac.ir/article-1-5093-en.html

[19] Rashedi V, Asadi-Lari M, Foroughan M, Delbari A, Fadayevatan R. Mental health and pain in older adults: findings from Urban HEART-2. Community Mental Health Journal. 2017; 53(6):719-24. [DOI:10.1007/s10597-017-0082-2] [PMID]

[20] Nabavi SH, Alipour F, Hejazi A, Rashedi V. [Relationship between social support and mental health in older adults (Persian)]. Medical Journal of Mashhad University of Medical Sciences. 2014 57(7):841-6. http://mjms.mums.ac.ir/article_3756.htm

[21] Alavi M, Jorjoran Shushtari Z, Noroozi M, Mohammadi Shahboulaghi F. [Mental health and related factors in old population in Tehran 2014-2015) (Persian)]. Journal of Mazandaran University of Medical Sciences. 2018; 27(158):112-22. http:/ /jmums.mazums. ac.ir/article-1-8866-en.html

[22] Nakano A. The relationship between mental health and selfrated health in older adults. Japan: Kobe University. 2014 http://www.econ.kobe-u.ac.jp/activity/publication/dp/ pdf/2014/1423.pdf

[23] Heine C, Browning CJ. Mental health and dual sensory loss in older adults: A systematic review. Frontiers in Aging Neuroscience. 2014; 6:83. [DOI:10.3389/fnagi.2014.00083] [PMID] [PMCID]

[24] Borhaninejad V, Rashedi V. COVID-19 Pandemic: Opportunity to advanced home care for oder adults. Journal of Gerontological Social Work. 2020; 63(6-7):629-30. [DOI:10.1080/01634372.2020.17 69790] [PMID]

[25] Drageset J, Dysvik E, Espehaug B, Natvig GK, Furnes B. Suffering and mental health among older people living in nursing homes-a mixed-methods study. Peer J. 2015; 3:e1120. [DOI:10.7717/peerj.1120] [PMID] [PMCID]

[26] Lahelma E, Laaksonen M, Martikainen P, Rahkonen O, SarlioLähteenkorva S. Multiple measures of socioeconomic circumstances and common mental disorders. Social Science \& Medicine. 2006; 63(5):1383-99. [DOI:10.1016/j.socscimed.2006.03.027] [PMID]

[27] Shojaei SF, Masoumi R. The importance of mental health training for psychologists in COVID-19 outbreak. Middle East Journal of Rehabilitation and Health Studies. 2020; 7(2):e102846. [DOI:10.5812/mejrh.102846]

[28] Huang X, Yang H, Wang HH, Qiu Y, Lai X, Zhou Z, et al. The association between physical activity, mental status, and socia and family support with five major non-communicable chronic diseases among elderly people: A cross-sectional study of a rural population in Southern China. International Journal of Environmental Research and Public Health. 2015; 12(10):13209-23. [DOI:10.3390/ijerph121013209] [PMID] [PMCID] 
[29] Kang MY, Kang YJ, Lee W, Yoon JH. Does long-term experience of nonstandard employment increase the incidence of depression in the elderly? Journal of Occupational Health. 2016; 58(3):247-54. [doi: 10.1539/joh.15-0169-OA]

[30] Tan ME, Sagayadevan V, Abdin E, Picco L, Vaingankar J, Chong SA, et al. Employment status among the $S$ ingapore elderly and its correlates. Psychogeriatrics. 2017; 17(3):155-63. [DOI:10.1111/psyg.12206] [PMID]

[31] Xinhuanet. Bat soup, biolab, crazy numbers...Misinformation "infodemic" on novel coronavirus exposed [Internet]. 2020 [Updated 2020 February 04]. Available from: http://www.xinhuanet.com/english/2020-02/04/c_138755586.htm

[32] Roth F, Brönnimann G. Focal report 8: Risk analysis using the internet for public risk communication. Center for Security Studies (CSS), ETH Zürich. 2013; (8). https:/ / css.ethz.ch/en/services/ digital-library/publications/publication.html/168875

[33] Gao J, Zheng P, Jia Y, Chen H, Mao Y, Chen S, et al. Mental health problems and social media exposure during COVID-19 outbreak. Plos One. 2020; 15(4):e0231924. [DOI:10.1371/journal. pone.0231924] [PMID] [PMCID]

[34] Rashedi V, Borhaninejad V. Age-based discrimination in Covid-19 patient care. Indian Journal of Medical Ethic. 2020; V(3):255. [DOI:10.20529/IJME.2020.74]

[35] Niederkrotenthaler T, Stack S, Till B, Sinyor M, Pirkis J, Garcia D, et al. Association of increased youth suicides in the United States with the release of 13 Reasons Why. JAMA Psychiatry. 2019; 76(9):933-40. [DOI:10.1001/jamapsychiatry.2019.0922] [PMID] [PMCID]

[36] Ji D, Ji YJ, Duan XZ, Li WG, Sun ZQ, Song XA, et al. Prevalence of psychological symptoms among Ebola survivors and healthcare workers during the 2014-2015 Ebola outbreak in Sierra Leone: A cross-sectional study. Oncotarget. 2017; 8(8):12784-91. [DOI:10.18632/oncotarget.14498] [PMID] [PMCID] 